\title{
Efficacy of bi-monthly hepatic arterial infusion chemotherapy for advanced hepatocellular carcinoma
}

\author{
Kei Moriya ${ }^{1}$, Tadashi Namisaki ${ }^{1}$, Shinya Sato ${ }^{1}$, Akitoshi Douhara ${ }^{1}$, Masanori Furukawa ${ }^{1}$, Hideto \\ Kawaratani $^{1}$, Kosuke Kaji ${ }^{1}$, Mitsuteru Kitade ${ }^{1}$, Naotaka Shimozato ${ }^{1}$, Yasuhiko Sawada ${ }^{1}$, Kenichiro Seki ${ }^{1}$, \\ Soichiro Saikawa ${ }^{1}$, Hiroaki Takaya ${ }^{1}$, Takemi Akahane ${ }^{1}$, Akira Mitoro', Yasushi Okura ${ }^{2}$, Junichi Yamao², \\ Hitoshi Yoshiji ${ }^{1}$
}

${ }^{1}$ Division of Gastroenterology and Hepatology, Third Department of Internal Medicine, ${ }^{2}$ Department of Endoscopy, Nara Medical University, Kashihara, Nara, Japan

Contributions: (I) Conception and design: K Moriya, J Yamao, H Yoshiji; (II) Administrative support: None; (III) Provision of study materials or patients: None; (IV) Collection and assembly of data: M Furukawa, H Kawaratani, K Kaji, M Kitade, N Shimozato, Y Sawada, K Seki, S Saikawa, H Takaya, T Akahane, A Mitoro, Y Okura; (V) Data analysis and interpretation: None; (VI) Manuscript writing: All authors; (VII) Final approval of manuscript: All authors.

Correspondence to: Kei Moriya. Division of Gastroenterology and Hepatology, Third Department of Internal Medicine, Nara Medical University, 840 Shijo-cho, Kashihara, Nara 634-8522, Japan. Email: moriyak@naramed-u.ac.jp.

Background: Even though the Barcelona Clinic Liver Cancer (BCLC) staging system is widely accepted, controversies on the management of hepatocellular carcinoma (HCC) still exist. We evaluated the efficacy of an approach with repeated hepatic arterial infusion chemotherapy (HAIC) given at eight-week intervals for the treatment of advanced HCC.

Methods: Of the 66 compensated cirrhotic patients with advanced HCC refractory to transcatheter arterial chemo-embolization (TACE) enrolled in our study, 21 were treated by bi-monthly hepatic arterial infusion chemotherapy (B-HAIC) and the rest by sorafenib. The overall survival periods, curative responses, and adverse events in each group were retrospectively analyzed.

Results: The efficacy rate was significantly higher in the B-HAIC group (38\%, $11 \%, \mathrm{P}<0.05)$. The median survival time and the survival rate at 12 months in the B-HAIC group were 567 days and $70.8 \%$, and those in the sorafenib group were 366 days and $47.6 \%$, respectively. Thus, our data suggests that the B-HAIC treatment is not inferior to sorafenib for the treatment of advanced HCC in compensated cirrhotic patients. Furthermore, the occurrence of serious adverse events leading to discontinuation of treatment was less frequent in the B-HAIC group.

Conclusions: Given the hepatic function reserve preservation afforded by the B-HAIC treatment in our experience, we suggest that B-HAIC should be considered an alternative strategy for advanced HCC patients who do not respond to TACE.

Keywords: Hepatocellular carcinoma (HCC); hepatic arterial infusion chemotherapy (HAIC); cisplatin; sorafenib; chemotherapy

Submitted Apr 04, 2018. Accepted for publication May 13, 2018.

doi: 10.21037/jgo.2018.05.13

View this article at: http://dx.doi.org/10.21037/jgo.2018.05.13

\section{Introduction}

According to the worldwide survey of World Health Organization in 2015, hepatic cancer (causing 0.79 million deaths per year) was the second-largest cause of cancer death, following lung cancer (1.69 million deaths per year) (http://www.who.int/mediacentre/factsheets/fs297/ en/, Accessed 22 Dec 2017). Hepatic cancer is clinically silent during its early stages, making it difficult to detect 
promptly unless regular mechanical examinations like ultrasonography, computed tomography (CT), or magnetic resonance imaging (MRI) are performed. The antitumor agent sorafenib, shown to be useful for advanced hepatocellular carcinoma (HCC) in 2008 (1), is the only established first-line chemotherapeutic agent. However, sorafenib affects the patient's hepatic functional reserve because of its metabolism (2), and the decision to use it requires prior appropriate assessments of hepatic functional reserve. Selective transcatheter arterial chemo-embolization (TACE) has been widely applied as a treatment for HCC but a large-scale randomized case control study proving its efficacy and superiority is lacking, and reports have shown a positive correlation between TACE treatment and the loss of hepatic functional reserve (3-5). Most patients with advanced HCC have limited normal hepatic function and concomitant disease, and their prognosis depends in part on their levels of hepatic functional reserve.

The 2013 clinical practice guidelines for HCC of the Japan Society of Hepatology (JSH) recommend chemotherapy with either hepatic arterial infusion chemotherapy (HAIC) or sorafenib for TACE refractory advanced HCC patients. But the knowledge as to which regimen is better or which one carries the longer median overall survival is lacking. HAIC allows for repetitive delivery of high intrahepatic drug concentrations without the need for synchronous embolization of the hepatic vasculature, and results in acceptable toxicity levels.

In this study, we hypothesized a bi-monthly HAIC protocol would be useful for treating patients with advanced HCC and we compared the approach with sorafenib therapy using data from two groups of patients. The median overall survival period of the HAIC group was almost 200 days longer than that of the sorafenib group. HAIC is simple, easy to manage, and widely available, and could be used in compensated cirrhotic patients.

\section{Methods}

\section{Patients}

To determine the best treatment for the patients with advanced HCC, the records of 96 chemo-naïve patients who were refractory to TACE or had distinct extrahepatic metastasis lesions admitted to the Nara Medical University Hospital between January 2009 and December 2014 were enrolled in this retrospective study. Twenty-one of the 46 cases treated with HAIC were enrolled in the bi-monthly hepatic arterial infusion chemotherapy (B-HAIC) group, after excluding 24 patients with decompensated cirrhosis and one patient who had died within four weeks after the beginning of treatment due to acute disease progression. And, 45 out of 50 cases treated with sorafenib were enrolled in the sorafenib group, after excluding five patients who had died within 4 weeks after the beginning of treatment or who had not been able to continue the sorafenib treatment for more than 2 weeks. The preceding TACE sessions had been carried out with an emulsion containing anticancer agents and lipiodol followed by gelatin sponge particles. TACE refractory cases were diagnosed according to the guidelines of the JSH and the Liver Cancer Study Group of Japan (LCSGJ) (6,7). For intrahepatic lesions, TACE failure was defined as $\geq 2$ consecutive ineffective responses of treated tumors (viable lesions $>50 \%$ ) or $\geq 2$ consecutive progressive increases in total tumor counts, despite a prior change in the choice of chemotherapeutic agent or reanalysis of the feeding artery. Ineffective responses were evaluated by CT/MRI 1-3 months after an adequately performed selective TACE procedure. Other TACE failure criteria included the continuous elevation of tumor marker levels immediately after TACE (although a transient minor decrease could have been observed) and the appearance of vascular invasion and extrahepatic spread. Diagnosis of HCC was based on the characteristic radiological images together with increases in the serum alpha-fetoprotein (AFP) and/or des-gamma carboxy prothrombin (DCP) levels. In our hospital, sorafenib is indicated for treating patients with highly advanced HCC whose hepatic functional reserves are classified as Child-Pugh class A. Informed consent forms of each treatment were obtained from the all patients before the initiation of treatment. The Ethics Committee of the Nara Medical University Hospital approved this study (approval \#000522), which was conducted in accordance with the ethical principles in the Japanese ethics guideline for epidemiological research [http://www.mhlw.go.jp/ file/06-Seisakujouhou-10600000-Daijinkanboukouseikagak uka/0000080278.pdf, Accessed 22 Dec 2017].

\section{B-HAIC treatments}

For B-HAIC using cisplatin, intra-arterial cisplatin at $65 \mathrm{mg} / \mathrm{m}^{2}$ was administered over 30 minutes via a catheter inserted into the right or left hepatic artery every 8 weeks, for up to 6 courses or until disease progression or unacceptable adverse events occurred. An infusion of 3,000 mL or more of extracellular fluid was administered on the day of B-HAIC, 


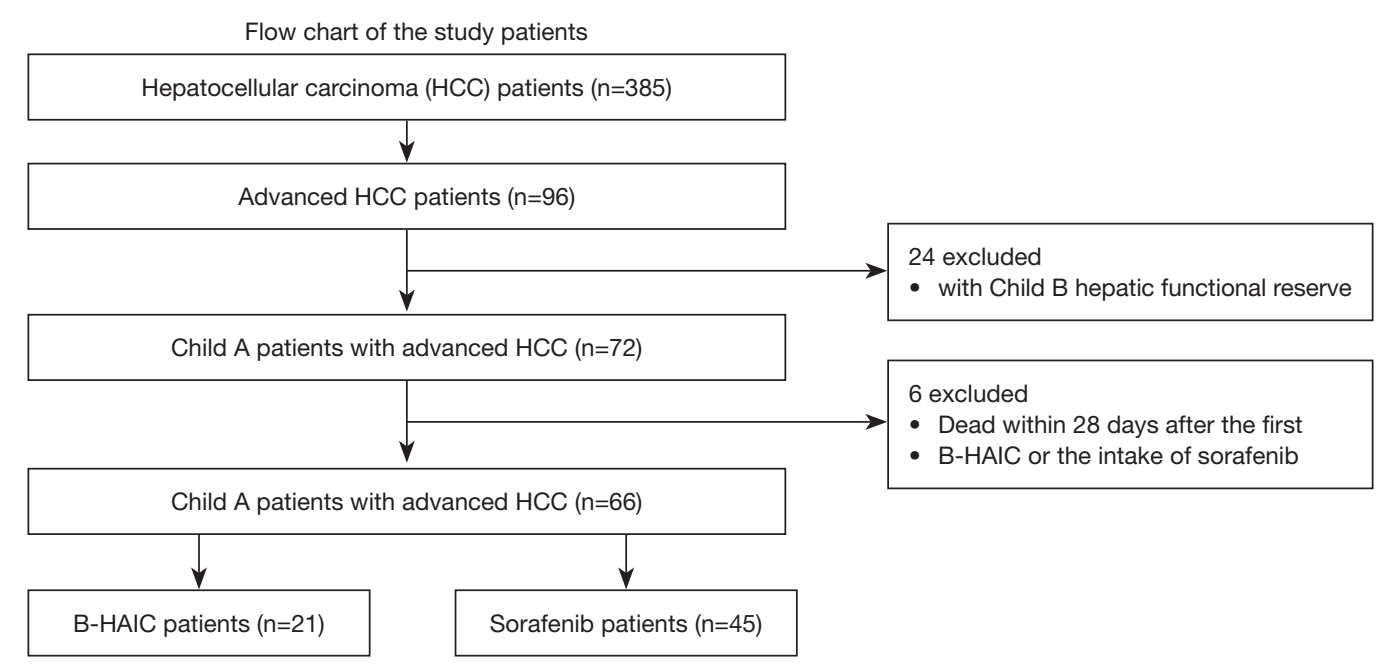

Figure 1 Flow chart of the patients. There were 385 initial patients' records with hepatocellular carcinoma of whom 66 with advanced hepatocellular carcinoma were included in the final analyses. These 66 patients were divided into the B-HAIC group and the sorafenib group. B-HAIC, bi-monthly hepatic arterial infusion chemotherapy.

and an infusion of at least $1,000 \mathrm{~mL}$ was continued on the next day in order to avoid renal toxicity effects of the drug. In case of inadequate urine output, diuretics were administered for several days. The patients who showed good response to the 6 B-HAIC courses were then treated with an implanted 5 -fluorouracil reservoir sequentially.

\section{Sorafenib treatment}

In this study, according to the widely accepted recommendation of JSH (2), sorafenib was prescribed only to the patients with advanced HCC whose hepatic functional reserves were classified as Child-Pugh class A. The starting dose of sorafenib for each case was decided during weekly group conferences considering the patient's clinical complications $(8,9)$. The treatment was continued as long as tolerated without disease progression. The doses were regularly readjusted depending on the severity of adverse events or the physical conditions as previously reported (10-14), and the maximum dose used went up to $800 \mathrm{mg} /$ day.

\section{Assessment and statistical analyses}

Dynamic enhanced CT or MRI examinations were used to confirm the anti-tumor effects every 1-2 months based on the modified Response Evaluation Criteria in Solid Tumors (RECIST) (15). The overall survival was calculated as the period from the day of starting treatment to the day of a patient's death or the final day of confirmed survival. The time to progression was defined as the period beginning on the first day of treatment until the day of the confirmation of tumor progression by radiological image examinations. The Chi-square test and Mann-Whitney U test were used to compare the patient characteristics and anti-tumor effect between B-HAIC and sorafenib treatment groups. We calculated the time to progression and overall survival using the Kaplan-Meier analysis, and compared differences between groups using the log-rank test. A $\mathrm{P}<0.05$ was considered as statistically significant. JMP version 11.2 (SAS Institute Inc.) software was used for statistical analyses.

\section{Results}

\section{Patient characteristics}

Of 385 patients with HCC in our hospital during the data generation period, only 96 patients with advanced HCC and who were refractory to TACE, who had undergone adequate radiological imaging assessments, and had sufficient blood tests were included in the study. We then decided to exclude 24 patients with decompensated cirrhosis and 6 patients as explained in the materials and methods section. We were left with the records of 21 patients treated with B-HAIC and 45 treated with sorafenib (Figure 1). The average interval between B-HAIC cycles was $1.9 \pm 0.4$ months and the average number of repeated cycles was $3.3 \pm 2.0$ times $($ mean $\pm \mathrm{SD})$. The total duration of B-HAIC treatment 
Table 1 Profiles of HCC patients with liver cirrhosis in this study $(\mathrm{n}=66)$

\begin{tabular}{lccc}
\hline Clinical profiles & B-HAIC $(\mathrm{n}=21)$ & Sorafenib $(\mathrm{n}=45)$ & $\mathrm{P}$ \\
\hline Age, median [range] (years) & $69[44-88]$ & $73[43-86]$ & 0.25 \\
Sex (male/female) & $16 / 5$ & $38 / 7$ & 0.41 \\
HCC numbers (1-3/4 and over) & $6 / 15$ & $12 / 33$ & 0.87 \\
Metastasis (with/without) & $1 / 20$ & $20 / 25$ & $<0.01$ \\
HCC clinical stage (II/III/IV) & $3 / 13 / 5$ & $1 / 10 / 34$ & $<0.01$ \\
T factor (T2/T3/T4) & $3 / 14 / 4$ & $4 / 21 / 20$ & 0.13 \\
intravascular invasion (with/without) & $10 / 11$ & $10 / 35$ & $<0.05$ \\
Preceding Tx. (with/without) & $21 / 0$ & $41 / 4$ & 0.39 \\
\hline
\end{tabular}

Categorical variables were tested with Fisher's exact test and continuous variables with Welch's two sample $t$-test.
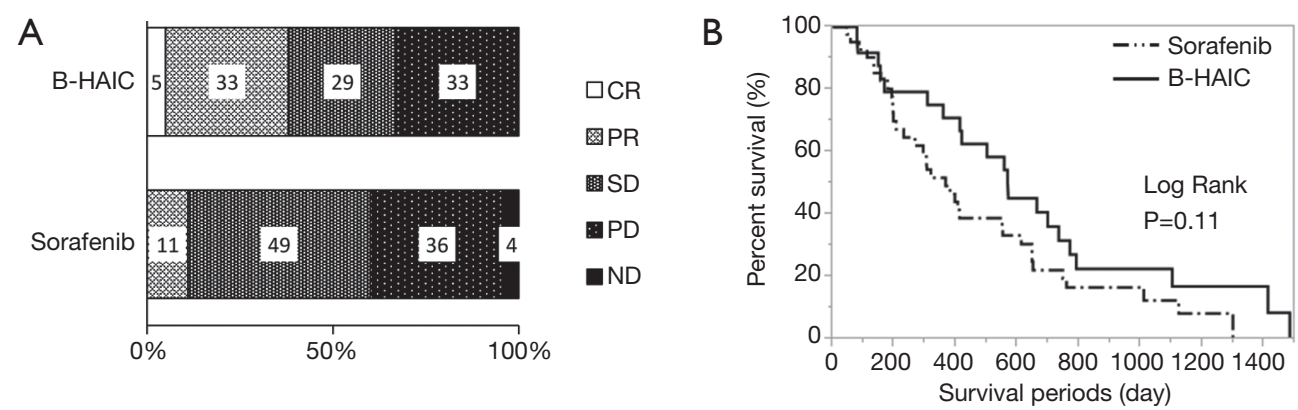

Figure 2 Chemopreventive effect of B-HAIC and sorafenib on advanced HCC. (A) The B-HAIC group included the more partial responder population, whereas the sorafenib group included the more stable disease rates; (B) Kaplan-Meier curves of overall survival of patients treated with B-HAIC (solid line) and sorafenib (dashed line). B-HAIC, bi-monthly hepatic arterial infusion chemotherapy; HCC, hepatocellular carcinoma.

averages $6.2 \pm 3.7$ months. According to the current guidelines for the appropriate use of sorafenib against HCC, this tablet should be prescribed only for the patients with compensated cirrhosis and chronic hepatitis (2), and the B-HAIC regimen can be also applied in patients with compensated cirrhosis. The profiles of patients with advanced HCC are shown in Table 1. The B-HAIC group patients with liver cirrhosis and Child-Pugh class A disease with advanced HCC $(n=21)$ had no extrahepatic metastasis (with one exception) and exhibited a lower rate of cases of advanced stage HCC at the beginning of the B-HAIC treatment. And, they exhibited a significantly higher rate of containing an intravascular tumor invasion.

\section{Efficacy and adverse events}

Compared with the patients in the B-HAIC group, more patients in the sorafenib group had extrahepatic metastases and advanced clinical stages of disease, and less of them could undergo additional treatments after the point of sorafenib failure. The best clinical response and overall survival rate for each treatment are shown in Figure 2A,B. The disease control rates (B-HAIC: $67 \%$, sorafenib: $60 \%$ ) were similar in both groups, but the efficacy rate was significantly higher in the B-HAIC group (38\% vs. $11 \%$, $\mathrm{P}<0.05)$. The median survival time and the survival rates at 6-, 12-, and 24-month were 567 days and $79.2 \%, 70.8 \%$, and $33.3 \%$, in the B-HAIC group patients, respectively; and 366 days, $73.8 \%, 47.6 \%$, and $19.1 \%$, respectively, in the sorafenib group patients. Also, the hepatic functional reserve in patients of the B-HAIC group was not changed during the treatment period, but that in the patients of the sorafenib group was worsened in the course of treatment (Figure $3 A$ ). We evidenced no detrimental renal function effects in patients of both groups (Figure 3B). The main reason for treatment discontinuance in the B-HAIC group 

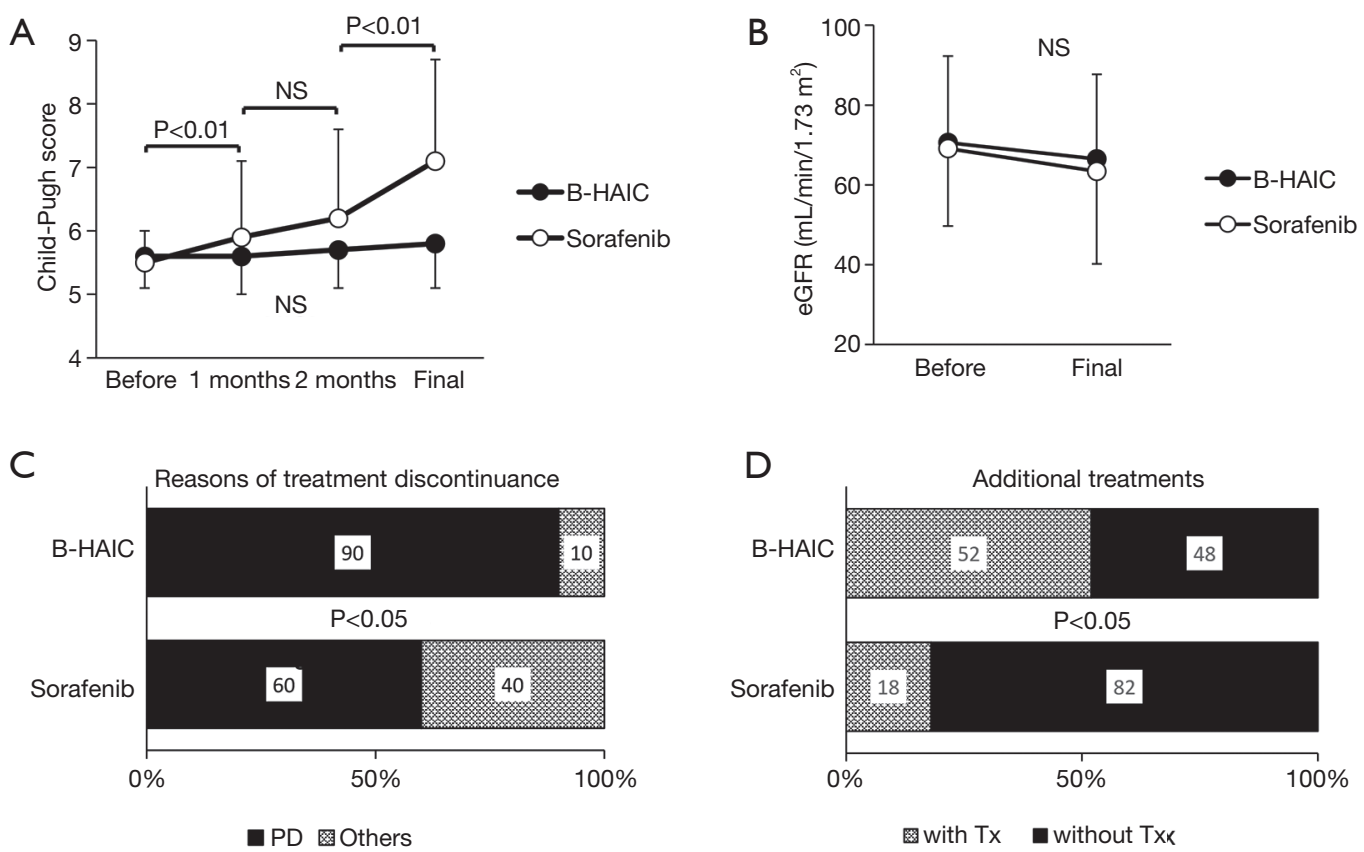

Figure 3 Adverse effect of B-HAIC and sorafenib on advanced HCC. (A,B) Changes in the Child-Pugh scores and eGFR before and after the treatments. The Child-Pugh score continuously increased during the medication of sorafenib. (C) Approximately half of the patients had to discontinue the use of sorafenib because of the reasons other than disease progression. (D) The B-HAIC group patients exhibited a higher rate of additional chemotherapy than the sorafenib group. B-HAIC, bi-monthly hepatic arterial infusion chemotherapy; HCC, hepatocellular carcinoma; eGFR, estimated glomerular filtration rate; NS, non-significant.

patients was disease progression (19 out of 21 cases: $90.4 \%$ ), whereas forty percent of patients (18 out of 45 cases) in the sorafenib group did not continue their treatment due to other reasons including physical or mental adverse events (Figure 3C). The B-HAIC group patients exhibited a higher rate of additional chemotherapy such as 5 -FU based regimen after the end of the B-HAIC treatments, when compared with patients in the sorafenib group $(n=45)$ whose hepatic functional reserves were classified into ChildPugh class A disease at the beginning of the treatment (Figure 3D).

In this retrospective cohort study, 20 of 66 cases were diagnosed as having advanced HCC with macroscopic intravascular and/or intrabiliary invasion and were treated by either B-HAIC or sorafenib. The clinical profiles of each group of patients are shown in Table S1. The treatment outcomes of each group including both the best curative responses and overall survival periods are shown in Figure $4 A, B$. The disease control rate of the B-HAIC group was actually same to that of the sorafenib group. However, in the group of patients treated for advanced HCC with intravascular invasion, no patient quit the B-HAIC therapy due adverse events, whereas four of ten patients (40\%) treated with sorafenib had to stop their treatment because of adverse events (including two cases of cerebral bleeding and two of severe general fatigue) (Figure 4C). In the subgroup analysis, more than a half of the B-HAIC group patients $(n=6)$ were successfully moved to an additional chemotherapy after the end of the B-HAIC treatments, whereas none of the sorafenib group patients had an additional treatment (Figure 4D).

\section{Influence on the patient's quality of life (QOL) and financial burden}

We also assessed the patients' QOL and found the B-HAIC, an inevitably invasive method, did not worsen the patients' QOL more than the sorafenib group (Figure S1A). Furthermore, we performed a simulation of medical costs, because clinicians will be expected to consider not only treatment efficacy but also cost-effectiveness. The estimated total medical costs associated with B-HAIC were similar to the costs of reservoir chemotherapy, and about half of the costs of the sorafenib regimen (Figure S1B). 

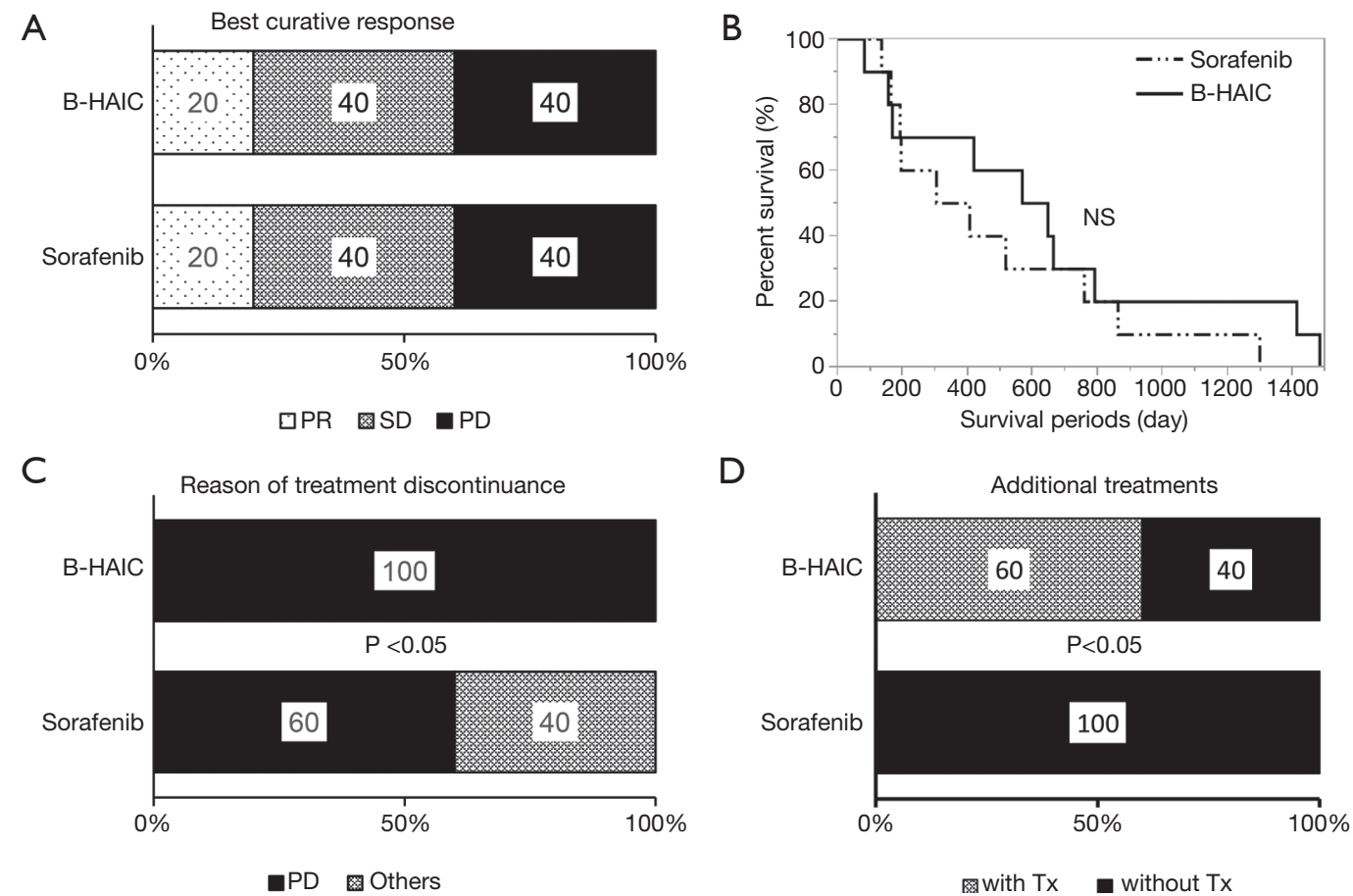

Figure 4 Chemopreventive effect of B-HAIC and sorafenib on advanced HCC with intravascular invasion. (A) Both of the B-HAIC group and the sorafenib group included the same populations of partial responder, stable disease, and progressive disease, respectively; (B) KaplanMeier curves of overall survival of patients treated with B-HAIC (solid line) and sorafenib (dashed line); (C) $40 \%$ of patients treated with sorafenib discontinued the treatment because of adverse events, whereas no patients terminated B-HAIC treatment due to adverse events; (D) the B-HAIC group patients exhibited a higher rate of additional chemotherapy than the sorafenib group. B-HAIC, bi-monthly hepatic arterial infusion chemotherapy; HCC, hepatocellular carcinoma; NS, non-significant.

\section{Discussion}

Numerous options for treatment to cure a patient's specific HCC case are available to clinicians, including surgical resections and radio frequency ablations, and several kinds of chemotherapeutic agents. Randomized control studies and two meta-analyses have found TACE (first established in Japan in 1978) to be more effective than the best supportive care (16-24). However, TACE can cause the loss of a patient's hepatic functional reserve (3-5), and theoretically lead to liver failure. And given, for example, the experience in cirrhosis patients whose life expectancy depends on their hepatic functional reserve $(25,26)$, maintaining a patient's hepatic functional reserve should be essential for any effective treatment for HCC.

HAIC is classified into two different types: in one a reservoir device is semi-permanently placed into the intrahepatic arterial space, and in the other (called B-HAIC) a vascular puncture for catheter manipulation is made for each infusion. The transient device is considered superior, because the activity of the fine-powder formulation of cisplatin used in the puncture infusion method appears to have only modest anti-tumor activity and can be conducted only every 4-6 weeks (27-29). However; the rate of complications of the transient device (hepatic artery obstruction, pseudo arterial aneurysm formation, and portrelated problems) are high at 43-56\% (30,31). Additionally, some of these complications could negatively impact a patient's hepatic functional reserve. For these reasons, personnel providing long-term repeated hepatic arterial chemotherapy infusions with a reservoir need to be wellversed with the technique.

B-HAIC is simple and not as invasive as the reservoir method, and we hypothesized it could be better for patients with relatively poor hepatic functional reserve or renal dysfunction. We demonstrated that the level of renal function and the hepatic functional reserve were not significantly decreased during the whole period of the B-HAIC in patients with HCC. Thus, we believe that non- 
invasiveness on a physical aspect of B-HAIC yielded the good results of overall survival period, and which were not at a disadvantage when compared with in the case of chemotherapy using reservoir.

Decompensated cirrhotic patients with advanced HCC are not considered good candidates for sorafenib therapy (2). For compensated cirrhotic patients, with HCC and refractory to TACE, sorafenib has shown efficacy (29), even though the drug has shown no efficacy as adjuvant therapy for preventing early recurrence after curative surgery for HCC (32). Nevertheless, the outcomes after either HAIC or sorafenib in patients with advanced HCC are still controversial (33-35).

In this retrospective cohort study, we demonstrated the efficacy of B-HAIC for cirrhotic patients with advanced HCC and for those with advanced HCC and portal venous tumor thrombosis (PVTT). The cirrhotic patients were effectively treated with B-HAIC without any serious adverse events. Even though our study included only a small group of patients with advanced HCC and PVTT, the disease control rate of B-HAIC and overall survival periods were similar to those of sorafenib, as has been published (30). From the viewpoint of maintaining hepatic functional reserve, the sequentially combined chemotherapeutic method which consists in initial administration of B-HAIC and sequential use of sorafenib, might generate favorable outcomes, which agree with the results of a clinical comparative study (36) and a recent randomized clinical phase II trial (37). Thus, we suggest that B-HAIC may be used as an alternative treatment modality to sorafenib in patients with advanced HCC and PVTT.

In reference to the Barcelona Clinic Liver Cancer (BCLC) staging system, a clinical network meta-analysis demonstrated that intra-arterial infusion chemotherapy is a superior treatment for patients at BCLC stage B and C, who are refractory to TACE and sorafenib (38).

As mentioned, our study is small, particularly when compared with other reports $(39,40)$ and it is retrospective so we could not take into account the prevalence of some clinical factors like age, gender, clinical stage of HCC, or sequential adjuvant chemotherapy. However, we actually regarded the hepatic functional reserve of cirrhotic patients as the most important factor in case of treating their HCC and narrowed the candidate of this study down to the patients with Child-Pugh class A disease.

In our hospital, the B-HAIC chemo-cycles are limited to a maximum of six in order to avoid serious adverse events, and this forced us to change the ongoing regimen even in cases where it had been absolutely effective. We followed B-HAIC chemo-cycles with a hepatic arterial infusion of cisplatin and 5-fluorouracil using reservoir devices.

Limitations notwithstanding, our study is the first to demonstrate the efficacy of bi-monthly HAIC for compensated cirrhotic patients classified as being ChildPugh class A. There have been several reports demonstrating the usefulness of reservoir chemotherapy for fluorouracilcisplatin regimens in cirrhotic patients with advanced HCC (30,31,39-43). The median survival periods in these reports range from 7.1 to 10.2 months, and none of them were better than the survival period of patients obtained with our B-HAIC method.

Thus, our results showing that B-HAIC preserves the hepatic function of these patients, lead us to suggest that B-HAIC should be considered an alternative strategy for advanced HCC patients who do not respond to TACE.

\section{Acknowledgements}

The study was supported in part by a grant-in-aid from Chugai Pharmaceutical and Yakult Honsha Co., and a grant-in-aid for research from Nara Medical University. Additionally, we thank Prof. Hiroshi Fukui for his kind and appropriate advice for completing this article.

\section{Footnote}

Conflicts of Interest: The authors have no conflicts of interest to declare.

Ethical Statement: The Ethics Committee of the Nara Medical University Hospital approved this study (approval \#000522). Informed consent forms of each treatment were obtained from the all patients before the initiation of treatment.

\section{References}

1. Llovet JM, Ricci S, Mazzaferro V, et al. SHARP Investigators Study Group. Sorafenib in advanced hepatocellular carcinoma. N Engl J Med 2008;359:378-90.

2. Kaneko S, Furuse J, Kudo M, et al. Guideline on the use of new anticancer drugs for the treatment of Hepatocellular Carcinoma 2010 update. Hepatol Res 2012;42:523-42.

3. Lu W, Li YH, Yu ZJ, et al. A comparative study of damage to liver function after TACE with use of low-dose versus conventional-dose of anticancer drugs in hepatocellular 
carcinoma. Hepatogastroenterology 2007;54:1499-502.

4. Nishikawa H, Osaki Y, Inuzuka T, et al. Branchedchain amino acid treatment before transcatheter arterial chemoembolization for hepatocellular carcinoma. World J Gastroenterol 2012;18:1379-84.

5. Ishikawa T, Kubota T, Horigome R, et al. Concurrent partial splenic embolization with transcatheter arterial chemoembolization for hepatocellular carcinoma can maintain hepatic functional reserve. Hepatol Res 2014;44:1056-61.

6. Kudo M, Izumi N, Kokudo N, et al. Management of hepatocellular carcinoma in Japan: Consensus-Based Clinical Practice Guidelines proposed by the Japan Society of Hepatology (JSH) 2010 updated version. Dig Dis 2011;29:339-64.

7. Kudo M, Matsui O, Izumi N, et al. Liver Cancer Study Group of Japan: JSH consensus-based clinical practice guideline for the management of hepatocellular carcinoma:2014 update by the Liver Cancer Study Group of Japan. Liver Cancer 2014;3:458-68.

8. Ishii T, Hatano E, Taura K, et al. Sorafenib in a hepatocellular carcinoma patient with end-stage renal failure: A pharmacokinetic study. Hepatol Res 2014;44:685-88.

9. Jo M, Yasui K, Kirishima T, et al. Efficacy and safety of sorafenib in very elderly patients aged 80 years and older with advanced hepatocellular carcinoma. Hepatol Res 2014;44:1329-38.

10. Morimoto M, Numata K, Kondo M, et al. Field practice study of half-dose sorafenib treatment on safety and efficacy for hepatocellular carcinoma: A propensity score analysis. Hepatol Res 2015;45:279-87.

11. Iavarone $M$, Cabibbo $G$, Piscaglia F, et al. SOFIA (SOraFenib Italian Assessment) study group. Field-practice study of sorafenib therapy for hepatocellular carcinoma: a prospective multicenter study in Italy. Hepatology 2011;54:2055-63.

12. Nishikawa H, Osaki Y, Endo M, et al. Japanese Red Cross Liver Study Group. Comparison of standard-dose and half dose sorafenib therapy on clinical outcome in patients with unresectable hepatocellular carcinoma in field practice: A propensity score matching analysis. Int J Oncol 2014;45:2295-302.

13. Kuzuya T, Ishigami M, Ishizu $Y$, et al. Fever within 2 Weeks of Sorafenib Therapy Predicts Favorable Treatment Efficacy in Patients with Advanced Hepatocellular Carcinoma. Oncology 2016;91:261-6.

14. Ogasawara S, Chiba T, Ooka Y, et al. Is intra-patient sorafenib dose re-escalation safe and tolerable in patients with advanced hepatocellular carcinoma? Int J Clin Oncol 2014;19:1029-36.

15. Lencioni R, Llovet JM. Modified RECIST (mRECIST) assessment for hepatocellular carcinoma. Semin Liver Dis 2010;30:52-60.

16. Lin DY, Liaw YF, Lee TY, et al. Hepatic arterial embolization in patients with unresectable hepatocellular carcinoma; a randomized controlled trial. Gastroenterology 1988;94:453-56.

17. Groupe d'Etude et de Traitement du Carcinome Hépatocellulaire. A comparison of lipiodol chemoembolization and conservative treatment for unresectable hepatocellular carcinoma. N Engl J Med 1995;332:1256-61.

18. Pelletier G, Ducreux M, Gay F, et al. Treatment of unresectable hepatocellular carcinoma with lipiodol chemoembolization: a multicenter randomized trial. Group CHC. J Hepatol 1998;29:129-34.

19. Bruix J, Llovet JM, Castells A, et al. Transarterial embolization versus symptomatic treatment in patients with advanced hepatocellular carcinoma: results of a randomized, controlled trial in a single institution. Hepatology 1998;27:1578-83.

20. Lo CM, Ngan H, Tso WK, et al. Randomized controlled trial of transarterial lipiodol chemoembolization for unresectable hepatocellular carcinoma. Hepatology 2002;35:1164-71.

21. Llovet JM, Real MI, Montaña X, et al. Arterial embolization or chemoembolization versus symptomatic treatment in patients with unresectable hepatocellular carcinoma: a randomized controlled trial. Lancet 2002;359:1734-39.

22. Doffoël M, Bonnetain F, Bouché O, et al. Multicenter randomised phase III trial comparing Tamoxifen alone or with Transarterial Lipiodol Chemoembolisation for unresectable hepatocellular carcinoma in cirrhotic patients (Fédération Francophone de Cancérologie Digestive 9402). Eur J Cancer 2008;44:528-38.

23. Cammà C, Schepis F, Orlando A, et al. Transarterial chemoembolization for unresectable hepatocellular carcinoma: meta-analysis of randomized controlled trials. Radiology 2002;224:47-54.

24. Llovet JM, Bruix J. Systematic review of randomized trials for unresectable hepatocellular carcinoma: Chemoembolization improves survival. Hepatology 2003;37:429-42.

25. Montano-Loza AJ. Clinical relevance of sarcopenia in 
patients with cirrhosis (review). World J Gastroenterol 2014;20:8061-71.

26. Pugh RN, Murray-Lyon IM, Dawson JL, et al. Transection of the oesophagus for bleeding oesophageal varices. Br J Surg 1973;60:646-9.

27. Iwasa S, Ikeda M, Okusaka T, et al. Transcatheter arterial infusion chemotherapy with a fine-powder formulation of cisplatin for advanced hepatocellular carcinoma refractory to transcatheter arterial chemoembolization. Jpn J Clin Oncol 2011;41:770-5.

28. Yoshikawa M, Ono N, Yodono H, et al. Phase II study of hepatic arterial infusion of a fine-powder formulation of cisplatin for advanced hepatocellular carcinoma. Hepatol Res 2008;38:474-83.

29. Ikeda M, Mitsunaga S, Shimizu S, et al. Efficacy of sorafenib in patients with hepatocellular carcinoma refractory to transcatheter arterial chemoembolization. J Gastroenterol 2014;49:932-40.

30. Song DS, Song MJ, Bae SH, et al. A comparative study between sorafenib and hepatic arterial infusion chemotherapy for advanced hepatocellular carcinoma with portal vein tumor thrombosis. J Gastroenterol 2015;50:445-54.

31. Oh MJ, Lee HJ, Lee SH. Efficacy and safety of hepatic arterial infusion chemotherapy for advanced hepatocellular carcinoma as first-line therapy. Clin Mol Hepatol 2013;19:288-99.

32. Wang SN, Chuang SC, Lee KT. Efficacy of sorafenib as adjuvant therapy to prevent early recurrence of hepatocellular carcinoma after curative surgery: A pilot study. Hepatol Res 2014;44:523-31.

33. Hatooka M, Kawaoka T, Aikata H, et al. Comparison of outcome of hepatic arterial infusion chemotherapy and sorafenib in patients with hepatocellular carcinoma refractory to transcatheter arterial chemoembolization. Anticancer Res 2016;36:3523-9.

34. Kawaoka T, Aikata H, Hyogo H, et al. Comparison of hepatic arterial infusion chemotherapy versus sorafenib

Cite this article as: Moriya K, Namisaki T, Sato S, Douhara A, Furukawa M, Kawaratani H, Kaji K, Kitade M, Shimozato N, Sawada Y, Seki K, Saikawa S, Takaya H, Akahane T, Mitoro A, Okura Y, Yamao J, Yoshiji H. Efficacy of bi-monthly hepatic arterial infusion chemotherapy for advanced hepatocellular carcinoma. J Gastrointest Oncol 2018;9(4):741-749. doi: 10.21037/jgo.2018.05.13 monotherapy in patients with advanced hepatocellular carcinoma. J Dig Dis 2015;16:505-12.

35. Fukubayashi K, Tanaka M, Izumi K, et al. Evaluation of sorafenib treatment and hepatic arterial infusion chemotherapy for advanced hepatocellular carcinoma: a comparative study using the propensity score matching method. Cancer Med 2015;4:1214-23.

36. Nagai H, Mukozu T, Ogino YU, et al. Sorafenib and hepatic arterial infusion chemotherapy for advanced hepatocellular carcinoma with portal vein tumor thrombus. Anticancer Res 2015;35:2269-77.

37. Ikeda M, Shimizu S, Sato T, et al. Sorafenib plus hepatic arterial infusion chemotherapy with cisplatin versus sorafenib for advanced hepatocellular carcinoma: randomized phase II trial. Ann Oncol 2016;27:2090-6.

38. Chang L, Wang Y, Zhang J, et al. The best strategy for HCC patients at each BCLC stage:a network meta-analysis of observational studies. Oncotarget 2017;8:20418-27.

39. Niizeki T, Sumie S, Torimura T, et al. Serum vascular endothelial growth factor as a predictor of response and survival in patients with advanced hepatocellular carcinoma undergoing hepatic arterial infusion chemotherapy. J Gastroenterol 2012;47:686-95.

40. Miyaki D, Aikata H, Honda Y, et al. Hepatic arterial infusion chemotherapy for advanced hepatocellular carcinoma according to Child-Pugh classification. J Gastroenterol Hepatol 2012;27:1850-7.

41. Tsai WL, Lai KH, Liang HL, et al. Hepatic arterial infusion chemotherapy for patients with huge unresectable hepatocellular carcinoma. PLoS One 2014;9:e92784.

42. Terashima T, Yamashita T, Arai K, et al. Feasibility and efficacy of hepatic arterial infusion chemotherapy for advanced hepatocellular carcinoma after sorafenib. Hepatol Res 2014;44:1179-85.

43. Shao YY, Liang PC, Wu YM, et al. A pilot study of hepatic arterial infusion of chemotherapy for patients with advanced hepatocellular carcinoma who have failed antiangiogenic therapy. Liver Int 2013;33:1413-9. 
Supplementary

Table S1 Profiles of HCC patients with intravascular invasion $(n=20)$

\begin{tabular}{lccc}
\hline Clinical profiles & B-HAIC $(\mathrm{n}=10)$ & Sorafenib $(\mathrm{n}=10)$ & $\mathrm{P}$ \\
\hline Age, median [range] (years) & $67[53-81]$ & $72[59-81]$ & 0.22 \\
Sex (male/female) & $9 / 1$ & $9 / 1$ & 1.00 \\
HCC numbers (1-3/4 and over) & $4 / 6$ & $2 / 8$ & 0.62 \\
Metastasis (with/without) & $1 / 9$ & $4 / 6$ & 0.30 \\
HCC clinical stage (III/IV) & $6 / 4$ & $6 / 4$ & 1.00 \\
\hline
\end{tabular}

Categorical variables were tested with Fisher's exact test and continuous variables with Welch's two sample t-test.
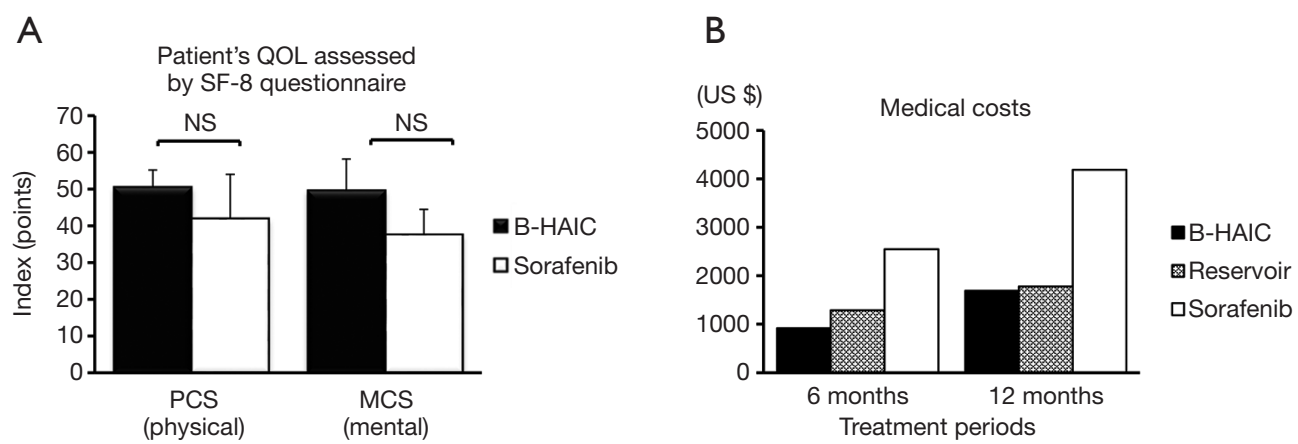

Figure S1 Patients' QOL and economic burden of each treatment. (A) B-HAIC had no more negative influence on the quality of life in terms of both physical and mental aspects; (B) the estimated medical costs of B-HAIC were almost less than half of the sorafenib costs. QOL, quality of life; B-HAIC, bi-monthly hepatic arterial infusion chemotherapy; NS, non-significant. 